\title{
Study on Selection of Heterogeneous Wireless Network in the View of Multi-Attribute Decision-Making
}

\author{
Longfei Guo*, Tingjie Lv, Tingting Li and Xia Chen
}

Key Laboratory of Information Management and Economy, Beijing University of Post and Telecommunication, Beijing, 100876, China

\begin{abstract}
Heterogeneous wireless network in the future will provide users with more service selections. The user can select the network with the best effect based on the specific demand and individual preference, and random selection will not only cause disorder of the network and low load efficiency but also impact the user's experience. The previous studies believe that the attribute of the network only has monotonous impact on the utility of user's use, namely, the larger the efficiency indicator the better, and the smaller the cost indicator the better. However, in reality, it changes with the conditions. The study divides the users into two types of quality preference and cost preference based on the model of multiattribute decision-making and constructs the network selection model in the environment of heterogeneous wireless network targeted at the voice business, video business and data business by taking into account the decision attributes of network bandwidth, delay, response speed, safety, cost to use, etc., thus realizing the objective of increasing the network utility of the user under the circumstance of adding no load to the network.
\end{abstract}

Keywords: Fuzzy mathematics, heterogeneous network, multi-attribute decision-making, network selection.

\section{INTRODUCTION}

Along with the development of wireless technology, the communications industry has witnessed earth-shaking changes. In the future, the network users will pursue better network convenience, mobility and network quality. These technologies satisfy the demand of the user for high-quality wireless network access; however, no network companies have ever been able to cover every corner or every user on the globe and various networks have different coverage scopes, response time, security coefficients, velocity, delay, access cost, etc. These will definitely lead to the wide integration of wireless heterogeneous networks.

To realize the integration of heterogeneous networks, the compatibility of networking protocol, compatibility of terminals and the optimal choice of multi-attribute should be solved first. The paper only discusses how the user makes the optimal choice among various wireless namely the problem of $\mathrm{ABC}$ (Always Best Connected). ABC is beneficial to the user, and the terminal can select networks, the most suitable network to access so as to adapt to the requirement of service application on network quality and avoid selecting blocking network, and predict the availability of network to avoid the sudden break of the network accessed; and the network selection and switching strategy made based on the type of user can lower the cost of user to access to the network. Meanwhile, $\mathrm{ABC}$ is also beneficial to the network supplier, because $\mathrm{ABC}$ can assist many networks in arranging the load between networks, thus increasing the maximum

*Address correspondence to this author at the No.10 Xitucheng Road, Haidian District, Beijing, 100876, P.R. China; Tel: 13810081769;

E-mail guolongf@bupt.edu.cn use ratio of network resources and further enhancing the business revenue of the operator.

With the purpose of accurately judging the effect of heterogeneous network selected, the study first makes standardization for the network attribute. Based on the previous studies, the paper proposes that the network attribute for the utility of user is not just in progressive increase or decrease. On this basis, the study selects the decision-making indicators of velocity, delay, jitter, packet loss probability and network cost, divides the businesses into voice business, video business and data business, and defines their own membership functions by the analytical method of fuzzy mathematics according to the requirement of all businesses on network attribute. When the user triggers network switching program, the user will transmit the network information collected to the fuzzy subsystems and obtain the utility value of the attribute.

Furthermore, through business division of the user and with analytic hierarchy process, the judgment matrix for network attribute and the test on the consistency of matrix are conducted, and weight of all attributes is finally obtained. According to the weighting coefficient of the network attribute and based on the simple linear weighting model, the network elimination factor and relative cost function are introduced to construct the multi-attribute decision-making model of heterogeneous network and obtain the utility of all networks for the user. The user can make their choice based on this, realizing multi-attribute decision-making.

\section{CURRENT STATUS OF RELEVANT STUDIES}

Network selection refers to the process of mobile terminal from one point of junction to another, therefore, it is also 


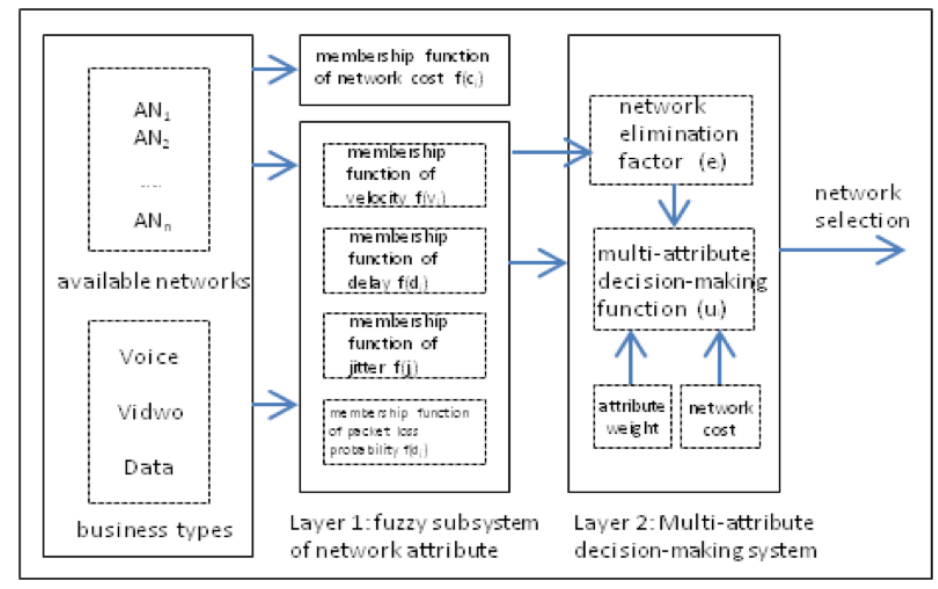

Fig. (1). Structure framework of the study.

called switching decision-making. The existing studies mainly divide the network switching process into three stages $[1$, 2]: 1) Collection stage of network information: collecting all information required to ensure whether the vertical switching program is triggered. 2) Switching decision-making stage: determining whether and how to execute the vertical switching by choosing suitable standard (such as user preference) and decision-making method, and sending the order to the execution stage, namely network selection or system selection stage. 3) Handover execution stage: making the mobile terminal access to the network newly selected based on the selection result provided in the previous stage. Therein, in the switching decision-making stage, the selection of decision-making indicator and switch decision-making strategy is the key for the whole network switching process, and also the key issue to be solved in the study.

First, decision-making indicator is the key factor to determine the realization of network switching. In the research model of network selection, no matter for the cost function or the utility function, generally the following four types of decision attributes need to be considered [3-6]: 1) Attribute relating to the network: coverage scope, network bandwidth, velocity, delay, link quality (RSS: received signal strength), CIR (Carrier-to Interferences Ratio), SIR (Signal-toInterferences Ratio), cost, safety level, etc.; 2) Attribute relating to terminal: terminal speed, electric quantity of battery, location information, etc.; 3) Attribute relating to user: personal data of user, user preference, etc.; 4) Attribute relating to service: service capability, service quality, etc.

In addition, there are many studies on the switching decision-making model based on RSS, cost/utility function decision-making, user orientation, multi-attribute decisionmaking, fuzzy logic, neural network and game theory. The study adopts multi-attribute decision-making model, including the following parts [7]: 1) Alternative schemes: optional schemes with limited quantity. The objective of the model is to select the optimal scheme finally through priority ordering. Alternative schemes can be policy, action, candidate, strategy, etc. The alternative scheme in the study is various heterogeneous networks available to access to the community of the user. 2) Multi-attribute: What the decision maker refers to when considering the alternative scheme is the attribute. In the model of heterogeneous network selection, the attribute can be related to the network, terminal, user or service. The paper emphasizes the velocity (throughput capacity), QoS value (time relay, blocking rate and packet loss probability of network) and network cost. 3) Decision matrix: In the format of matrix, the multi-attribute value of all alternative schemes is simply presented. In the decision matrix, $x_{i j}$ is the numerical value of No. $j$ attribute in No. $i$ alternative scheme. 4) Weight of attribute: Different decision makers stress different attributes while ordering the alternative schemes, so the weight of different attributes must be given to represent the importance degree of the attribute.

The study first establishes the fuzzy subsystems of decision attribute and makes standardization for various decision attributes through the membership function of the layer. Then, based on the weight coefficient of the attribute and with the utility function of multi-attribute decision-making, the utility of the network for the user is obtained, realizing the efficient network selection.

\section{FUZZY SUBSYSTEM OF NETWORK DECISION ATTRIBUTE}

The models in the paper have two parts: one is the fuzzy subsystem of network decision attribute and the other is the multi-attribute decision-making system, shown in Fig. (1).

Note: 1) Pay attention to three types of network businesses: Voice business, video business and data business. With different requirements of different businesses on the network attribute, in the network attribute subsystem of the model, the membership function changes with the business type of the user, thus only paying attention to the three types of core businesses. 2) Pay attention to five network decision attributes: The user makes comprehensive selection of network based on various attribute values, thus the core network decision attributes of velocity, delay of network, network's jitter (delay between networks), data packet loss probability and network cost are selected according to the perceptibility of the user for different businesses.

In the selection process of heterogeneous wireless network, the selection standard of the user is related to the business that the user currently applies. The high attribute value of network does not mean the high utility of the user, shown in Fig. (2) [8]. 


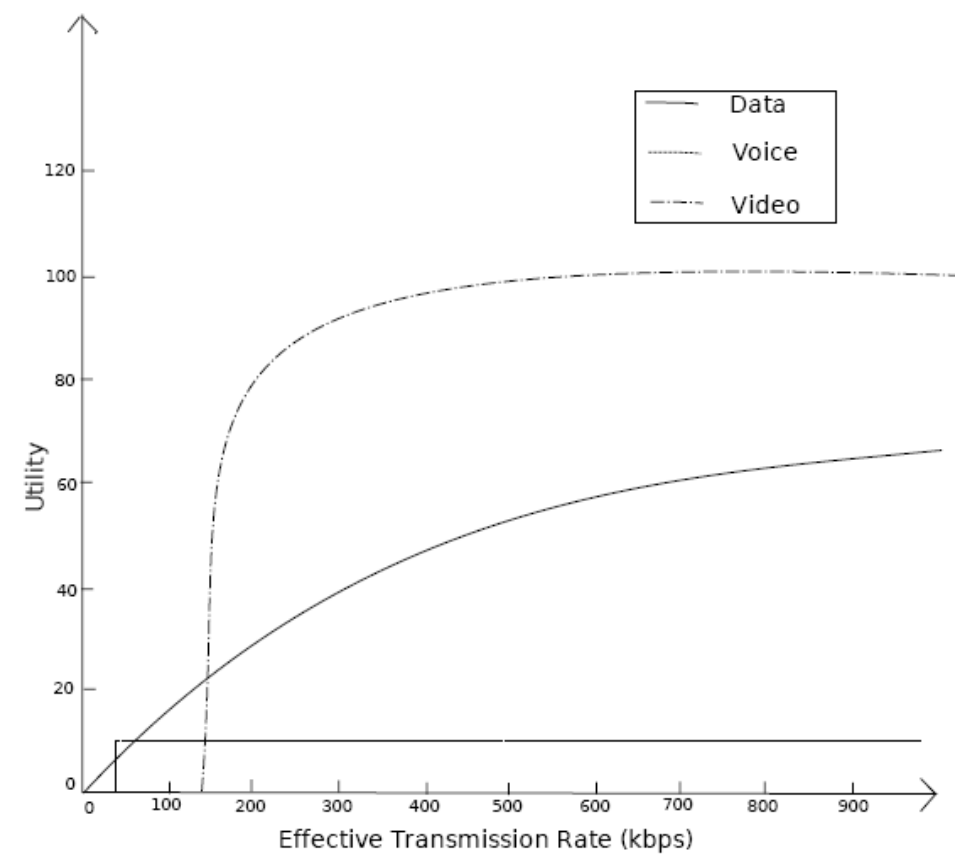

Fig. (2). Utility functions of voice, video and data businesses.

For voice business, when the data velocity is less than $13 \mathrm{kbps}$, the utility of the network for the user is 0 ; when the velocity of the network is no less than $13 \mathrm{kbps}$, the utility of the network for the user is 12 . For the video business, when the data velocity is less than $128 \mathrm{kbps}$, the utility of the network for the user is 0 ; when the velocity of the network is no less than $384 \mathrm{kbps}$, the utility of the network for the user is 100 ; and when the velocity is $128-384 \mathrm{kbps}$, the utility of the network for the user gradually increases. And for data business, the utility of the network for the user increases along with the transmission velocity.

The previous studies all presume that the greater the attribute value of the network, the greater the utility of the network. The analysis finds that for different businesses, the attribute of the network does not show monotone increasing with the utility of the user. Namely, high attribute value of the network does not represent greater utility of the user, and some attributes even show negative correlation with the utility. Based on this, the study takes into account the network cost and introduces the analytical method of membership function in the fuzzy mathematics based on the requirements of different business types for the attribute value.

Take five attributes of the network as five domains: velocity of network, delay, jitter, packet loss probability and network cost with domains of $\mathrm{V}=\{\mathrm{vi}|\mathrm{vi}\rangle=0\}$, (unit: kbps), $\mathrm{D}=\{$ di $\mid \mathrm{di}>=0\}$ (unit: $\mathrm{ms}$ ), $\mathrm{J}=\{\mathrm{ji}|\mathrm{ji}\rangle=0\}$ (unit: $\mathrm{ms}$ ), $\mathrm{L}=\{\mathrm{li}$ $\mid \mathrm{li}>=0\}$ (unit: 106) and $\mathrm{C}=\{\mathrm{ci} \mid \mathrm{ci}>=0\}$ (unit: single byte price) respectively. And the membership functions of the user utility to fuzzy sets in these domains are defined as $\mathrm{f}(\mathrm{v})$, $f(d), f(j), f(l)$ and $f(c)$ respectively.

Meanwhile, the study also defines that when the attribute value of the network can totally satisfy the requirement of the business for velocity and QoS, the utility of the user is 1 . Namely, when the attribute value of the network reaches a certain number and the user's experience cannot be better improved, the utility of the user is 1 . Correspondingly, it defines that when the network cannot fully satisfy the maximum value required by the business for velocity and QoS, the utility is 0 . The numbers between $0-1$ represent the degrees of membership of the network attribute for the utility of the user of 1 . The greater the degree of membership, the greater the utility.

According to the existing studies [8][9] and Fig. (2), it can be known that:

1), With respect to the requirements of voice, video and data business for QoS

2), the packet loss probability of voice shall not exceed $1 \%$, one-way delay shall not exceed $150 \mathrm{~ms}$, and the average one-way jitter shall aim for less than $30 \mathrm{~ms}$. The quality of voice business is directly impacted by all QoS factors. The packet loss probability of video shall not exceed 3\%, and the average one-way jitter shall aim for less than $100 \mathrm{~ms}$. It is shown in Table 1:

2) The utility of attribute value of network is not monotonous [9], and presents new algorithm aimed at the disadvantage of ELECTRE algorithm which can classify alternative networks (excluding strict inferior solution) but not provide complete ordering scheme. The study introduces reference attribute vector (the vector has the attribute value of the matching network required by the service - not the larger the better, it is only required to match the service), obtains the difference between the actual attribute value of network and the reference vector, and gets the absolute value for the acquisition of new decision matrix. The study holds that the less the matrix deviates from the attribute of the matching network, the better the corresponding network is (the more the attribute is, the more it deviates from the optimal choice), as shown in Fig. (3). 
Table 1. The upper and lower bounds of requirements of each business for velocity and QoS.

\begin{tabular}{|c|c|c|c|c|}
\hline \multirow{2}{*}{ Business Type } & \multicolumn{2}{|c|}{ Velocity (kbps) } & \multicolumn{2}{|c|}{ Delay (ms) } \\
\hline & Upper Bound & Lower Bound & Upper Bound & Lower Bound \\
\hline Voice & $>=13$ & $<13$ & 100 & 150 \\
\hline Video & 384 & 128 & 400 & - \\
\hline Data & - & 0 & 1000 & - \\
\hline \multirow{2}{*}{ Business type } & \multicolumn{2}{|c|}{ Jitter (ms) } & \multicolumn{2}{|c|}{ Packet loss probability $\left(/ 10^{6}\right)$} \\
\hline & Upper bound & Lower bound & Upper bound & Lower bound \\
\hline Voice & 15 & 30 & 15 & 10000 \\
\hline Video & 50 & 100 & 50 & 30000 \\
\hline Data & 100 & - & 100 & - \\
\hline
\end{tabular}

Note: The corresponding user utility of the attribute value of "upper bound" is 1 , and the corresponding user utility of the attribute value of "lower bound" is 0 .

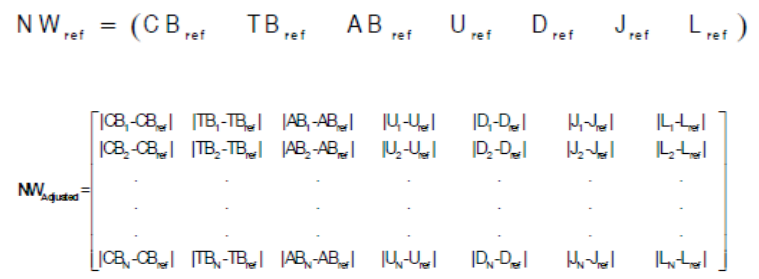

Fig. (3). Network parameter matrix with the introduction of reference attribute vector.

Though the study puts forward that the utility of the attribute is not monotonous; however, the method that new judgment matrix is obtained through simple introduction of reference attribute vector and elevation of difference between it and the actual network attribute may result in: When there are two networks with attribute values respectively greater than or less than the reference attribute vector, user may make wrong choices because the value of the network less than the reference attribute vector deviates less from the reference attribute during the comparison between the two networks.

3) International Telecommunications Union divides businesses into voice, video, data and background application programs. Therein, voice is divided into conversation class, message class and stream class; video is divided into video call, unilateral video, etc.; data are divided into web browsing, static picture, instant message, batch data, etc; background application programs are divided into fax, download link, etc. It also points out different businesses have different requirements for QoS, as shown in Fig. (4).

On the basis of the above analysis and with the analogue function of matlab simulation software as reference, the membership function of each network attribute can be obtained, and the utility function of each network attribute can be determined finally.

\subsection{Voice Business}

On the basis of the membership functions of velocity and QoS and with the analogue function of matlab simulation software as reference, the membership functions of its velocity, delay, jitter and packet loss probability are respectively defined as $f\left(v_{i}\right), f\left(d_{i}\right), f\left(j_{i}\right)$ and $f\left(l_{i}\right)$ :

$$
\begin{aligned}
& \mathrm{f}\left(v_{i}\right)=\left\{\begin{array}{l}
0, v<13 \\
1, v \geq 13
\end{array}\right. \\
& \mathrm{f}\left(d_{i}\right)=\left\{\begin{array}{r}
1, d \leq 100 \\
(150-d) /(150-100), 100<d<150 \\
0, d \geq 150
\end{array}\right. \\
& \mathrm{f}\left(j_{i}\right)=\left\{\begin{array}{c}
(30-j) /(30-15), 15<j<30 \\
0, j \geq 30 \quad 1, j \leq 15
\end{array}\right. \\
& \mathrm{f}\left(l_{i}\right)=\left\{\begin{array}{c}
\left(10^{4}-j\right) /\left(10^{4}-15\right), 15<j<10^{4} \\
0, j \geq 10^{4}
\end{array}\right.
\end{aligned}
$$

\subsection{Video Business}

It can be obtained by the same method as above:

$$
\begin{aligned}
& \mathrm{f}\left(v_{i}\right)=\left\{\begin{array}{r}
0, v<128 \\
(v-128) /(384-128), 128<v<384 \\
1, v \geq 384
\end{array}\right. \\
& \mathrm{f}\left(d_{i}\right)=\left\{\begin{array}{c}
1, d \leq 400 \\
1-\left(\frac{d-400}{d+400}\right)^{2}, d>400
\end{array}\right.
\end{aligned}
$$

$\mathrm{f}\left(j_{i}\right)=\left\{\begin{array}{c}1, j \leq 50 \\ (100-j) /(100-50), 50<j<100 \\ 0, j \geq 100\end{array}\right.$

$\mathrm{f}\left(l_{i}\right)=\left\{\begin{array}{c}1, j \leq 50 \\ \left(3 * 10^{4}-j\right) /\left(3 * 10^{4}-50\right), 50<j<3 * 10^{4} \\ 0, j \geq 3 * 10^{4}\end{array}\right.$

\subsection{Data Business}

It can be obtained by the same method as above:

$\mathrm{f}\left(v_{i}\right)=\left\{\frac{v}{v+200}, v \geq 0\right.$

$\mathrm{f}\left(d_{i}\right)=\left\{\begin{array}{c}1, d \leq 1000 \\ 1-\left(\frac{d-1000}{d+1000}\right)^{2}, d>1000\end{array}\right.$

$\mathrm{f}\left(j_{i}\right)=\left\{\begin{array}{c}1, j \leq 100 \\ 1-\left(\frac{j-100}{j+100}\right)^{2}, j>100\end{array}\right.$ 


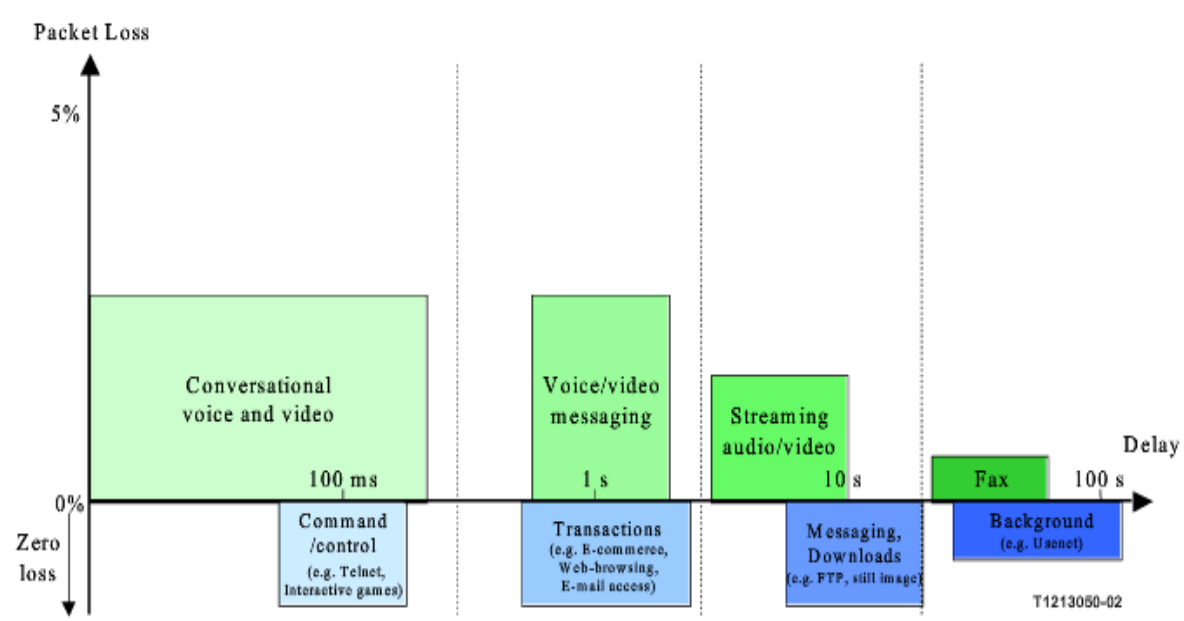

Fig. (4). User-centered QoS requirement reflection.

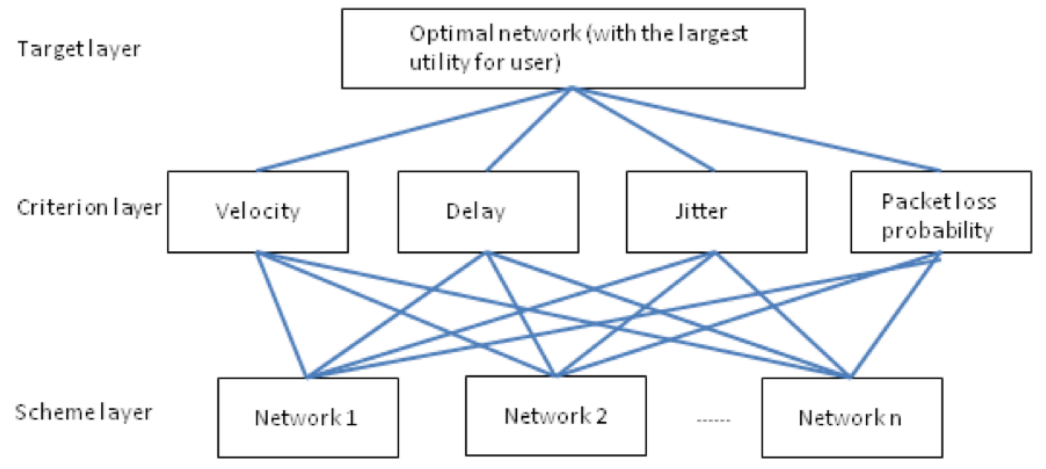

Fig. (5). AHP model of network selection.

$\mathrm{f}\left(l_{i}\right)=\left\{\begin{array}{c}1, l \leq 100 \\ 1-\left(\frac{l-1000}{l+1000}\right)^{2}, l>100\end{array}\right.$

\subsection{Cost Function}

By means of fully considering the preference for cost of user, users are divided into price preference type and quality preference type. On this basis, cost function is set:

In the formula, $p_{i}$ is the cost of a single byte of each network, and $\mathrm{n}$ represents $\mathrm{n}$ alternative networks. Thus, the cost function defined in the study is a relative cost, and is the proportion of the price of a certain network to the highest cost among all network costs [5]. $\alpha$ stands for the type of user; when the user is a quality preference type, $\alpha=0$; when the user is a price preference type, $\alpha=1$. This function is part of the multi-attribute decision-making function of the study.

\subsection{Network Elimination Factor}

The elimination factor of a network is denoted by and $n$ represents the number of the alternative networks. $\gamma_{i}$, 其中 $\mathrm{i}=1,2 \ldots \mathrm{n}, \gamma_{\mathrm{i}}=0$ 或 1 ,

When the utility of a network attribute for user of a network is 0 , and the user will not consider the network during the process of network selection. When the utility of all the network attributes for user of a network is greater than 0 .
This function is part of the multi-attribute decision-making function of the study.

\section{CONSTRUCTION OF MULTI-ATTRIBUTE DECI- SION-MAKING MODEL}

Through the membership functions in the system at the above layer, different attribute values are translated into the utility for user, namely, standard numerical values; the standard numerical values are transmitted to the multi-attribute decision-making, and the utility value of each network can be obtained through multi-attribute decision-making function; finally, according to the order of the each utility value, the most suitable network access can be selected.

\subsection{Weight Calculation of Network Attribute}

As shown in Fig. (5), the study adopts Analytic Hierarchy Process (AHP) to calculate each attribute weight. First, the network selection is layered:

Each value in the judgment matrix represents the importance degree of a factor in this layer compared to another factor with the upper layer as the criterion. The criterion layer of the study has four attributes, so four judgment matrixes will be constructed as shown in Table 2 :

In the matrix, represents the importance degree of compared to with respect to the upper layer - selection of optimal network; for example, $a_{23}$ represents the importance 
Table 2. Judgment matrix of network selection.

\begin{tabular}{|c|c|c|c|c|c|}
\hline \multicolumn{2}{|c|}{ Optimal Network } & Velocity $a_{1}$ & Delay $a_{2}$ & Jitter $a_{3}$ & Packet Loss Probability $a_{4}$ \\
\hline \multicolumn{6}{|c|}{ Velocity $a_{1}$} \\
\hline \multicolumn{6}{|c|}{ Delay $a_{2}$} \\
\hline \multicolumn{6}{|c|}{ Jitter $a_{3}$} \\
\hline \multicolumn{6}{|c|}{ Packet loss probability $a_{4}$} \\
\hline \multirow{5}{*}{$\omega_{\text {voice }}=$} & & Velocity & Delay & Jitter & Packet loss probability \\
\hline & Velocity & 1 & $1 / 7$ & $1 / 7$ & $1 / 3$ \\
\hline & Delay & $7 / 1$ & 1 & $5 / 4$ & $3 / 1$ \\
\hline & Jitter & $7 / 1$ & $4 / 5$ & 1 & $3 / 1$ \\
\hline & Packet loss probability & $3 / 1$ & $1 / 3$ & $1 / 3$ & 1 \\
\hline \multirow{5}{*}{$\omega_{\text {video }}=$} & & Velocity & Delay & Jitter & Packet loss probability \\
\hline & Velocity & 1 & $7 / 1$ & $2 / 1$ & $3 / 2$ \\
\hline & Delay & $1 / 7$ & 1 & $1 / 4$ & $1 / 5$ \\
\hline & Jitter & $1 / 2$ & $4 / 1$ & 1 & $2 / 3$ \\
\hline & Packet loss probability & $2 / 3$ & $5 / 1$ & $3 / 2$ & 1 \\
\hline \multirow{5}{*}{$\omega_{\text {video }}=$} & & Velocity & Delay & Jitter & Packet loss probability \\
\hline & Velocity & 1 & $5 / 1$ & $3 / 1$ & $4 / 5$ \\
\hline & Delay & $1 / 5$ & 1 & $2 / 5$ & $1 / 6$ \\
\hline & Jitter & $1 / 3$ & $5 / 2$ & 1 & $1 / 3$ \\
\hline & Packet loss probability & $4 / 5$ & $6 / 1$ & $3 / 1$ & 1 \\
\hline
\end{tabular}

Table 3. Weight of each network attribute of different businesses.

\begin{tabular}{|c|c|c|c|c|}
\hline Business & Velocity & Delay & Jitter & Packet Loss Probability \\
\hline \hline Voice & 0.053 & 0.445 & 0.371 & 0.131 \\
\hline Video & 0.428 & 0.058 & 0.215 & 0.3 \\
\hline Data & 0.382 & 0.07 & 0.149 & 0.399 \\
\hline
\end{tabular}

degree of factor compared to the jitter of factor. The importance is represented by judgment scale, and the scale covers numbers 1 9. From small to larger, 1 9 represent the increasing degrees of importance.

In consideration of various characteristics of voice, data and video businesses, these businesses have different requirements for network attribute. By virtue of vast collections and suggestions from experts, the following judgment matrixes are obtained.

After the check of consistency of matrixes, the weight of each network attribute of each type of business is acquired, as shown in the Table $\mathbf{3}$.

\subsection{Multi-Attribute Decision-Making Function - Utility Function}

The decision-making process of heterogeneous network selection requires consideration of multiplefactors, which just conforms to the principle of multi-attribute decisionmaking. Analysis is conducted by means of linear weighting, and its principle model is as follows:

Therein, represents the measured value of alternative scheme; represents the index value of standardized layer; represents the weighted value of each evaluation index; and $\mathrm{n}$ represents the number of the evaluation index.

On the basis of linear weighting function, the study takes into account user's preference for price and defines the utility function for the study; that is, the utility value for user = the utility value the user obtains from the network parameter - cost. The formula is a general linear weighting function. However, if the utility of an attribute value of the network for user is 0 , user will not consider the network during the process of heterogeneous network selection. Hence, as for the network elimination factor: 
And is the relative price function of the third chapter. The study classifies users into cost-preference type and qualitypreference type. When the user is a price-preference type; when the user is a quality-preference type.

In utility function, must be standardized attribute value. The standardization handling method of this study is each membership function in the third chapter, which makes the network attribute value for user fuzzy and changes it to numerical value of $0 \sim 1$ as the utility for user. In the formula, and $\bar{v}_{i}=\mathrm{f}\left(v_{i}\right)$.

Thus, the multi-attribute decision-making utility function of heterogeneous wireless network is:

$$
\begin{aligned}
& u_{i}=\gamma_{\mathrm{i}}\left(\omega_{1} * \mathrm{f}\left(v_{i}\right)+\omega_{2} * \mathrm{f}\left(d_{i}\right)+\omega_{3} * \mathrm{f}\left(j_{i}\right)+\omega_{4} * \mathrm{f}\left(l_{i}\right)\right)+ \\
& \alpha *\left(\mathrm{p}_{\mathrm{i}} / \operatorname{maxp}_{\mathrm{i}^{\prime}}\right) \mathrm{i}=1,2 \ldots \mathrm{n},
\end{aligned}
$$

User will select networks with largest utility for switch:

$$
u_{\text {best }}=\operatorname{argmax}\left(u_{i}\right) \mathrm{i}=1,2 \ldots \mathrm{n}
$$

The start of the process of network selection can only be conducted after triggering the network selection, so as to try to avoid user's frequent switch between networks and lower network load. The following four scenarios may trigger network switch:

$\diamond \quad$ The user leaves the coverage area of current network;

$\diamond \quad$ The attribute related to service is degraded (network parameter declines);

$\diamond \quad$ The user' preference has changed;

$\diamond \quad$ According to the attributes related to cost and application, there is better alternative operation.

\section{SUMMARY AND PROSPECT}

The study discusses the network selection of heterogeneous wireless network, and divides the network selection algorithm into two layers, namely, fuzzy handling of network attribute and construction of multi-attribute decision-making model. Based on the previous studies, the study divides businesses into voice business, video business and data business, classifies users into quality-preference type and pricepreference type, introduces network elimination factor, relative cost function and other influencing factors, and defines the decision-making function of the study on the basis of multi-attribute decision-making function. The result of the study is closer to actual application scenarios, and its indepth exploration of heterogeneous wireless network selec- tion and bold attempt will offer reference for the future network development.

In order to simplify the complexity of the research question, the study selects three types of businesses and five decision-making attributes of network; in the future research, an attempt to the optimal selection of network under complex conditions can be made.

\section{CONFLICT OF INTEREST}

The authors confirm that this article content has no conflict of interest.

\section{ACKNOWLEDGEMENTS}

This Project was supported by National Basic Research Program of China (973 Program) under Grant No. 2012CB315805; National Natural Science Foundation of China under Grant No.71172135 and No.71231002; "The Fundamental Research Funds for the Central Universities"(2013RC0603).

\section{REFERENCES}

[1] P. Chan, R. Sheriff, Y. Hu, P. Conforto, and C. Tocci, "Mobility management incorporating fuzzy logic for a heterogeneous IP environment", IEEE Communications Magazine, vol. 39, no. 12, pp. $42-51,2001$.

[2] E. Stevens-Navarro, and V. Wong, "Comparison between vertical handoff decision algorithms for heterogeneous wireless networks", Proceedings of IEE Vehicular Technology Conference (VTC-Spring), vol. 2, pp. 947-951, 2006.

[3] X. Cai, L. Chen, R. Sofia, and Y. Wu, "Dynamic and user-centric network selection in heterogeneous networks", Proceeding of IEEE International Performance Computing and Communications Conference IPCCC (IPCCC), pp. 538-544, 2007.

[4] F. Bari, and V. C. M. Leung, "Automated network selection in a heterogeneous wireless network environment", IEEE Network, vol. 21, pp.34-40, 2007.

[5] E. Stevens-Navarro, and V. W. S. Wong, "Comparison between vertical handoff decision algorithms for heterogeneous wireless networks", Proceeding of IEEE Vehicular Technology Conference (VTC Spring), pp. 947-951, 2006.

[6] Q. Song, and A. Jamalipour, "Network selection in an integrated wireless LAN and UMTS environment using mathematical modeling and computing techniques", IEEE Wireless Communications, vol. 12, pp. 42-48, 2005.

[7] C. L. Hwang, and K. Yoon, "Multiple attribute decision making methods and applications", Springer-Verlag 1981.

[8] M. A. Khan, U. Toseef, S. Marx, and C. Goerg, "Auction based Interface Selection with Media Independent Handover Services and Flow Management", European Wireless Conference, pp. 429-436, 2010.

[9] F. Bari, and V. Leung, "Application of ELECTRE to network selection in a heterogeneous wireless network environment", Processing of IEEE Wireless Communications and Networking Conference (WCNC), pp. 3810-3815, 2007.

\footnotetext{
Received: September 16, 2014

Revised: December 23, 2014

Accepted: December 31, 2014

(c) Guo et al.; Licensee Bentham Open.

This is an open access article licensed under the terms of the (https://creativecommons.org/licenses/by/4.0/legalcode), which permits unrestricted, noncommercial use, distribution and reproduction in any medium, provided the work is properly cited.
} 